\title{
Aspectos Afetivos e o Desempenho Acadêmico de Escolares ${ }^{1}$
}

\author{
Adriana Vilela Jacob², Sonia Regina Loureiro, \\ Edna Maria Marturano, Maria Beatriz M. Linhares e \\ Vera Lúcia Sobral Machado \\ Universidade de São Paulo, Ribeirão Preto
}

\begin{abstract}
RESUMO - Objetivou-se caracterizar através do Desenho da Casa-Árvore-Pessoa (HTP) e do Teste das Pirâmides Coloridas de Pfister (TPC) o funcionamento afetivo de 50 crianças com idade entre 8 e 12 anos, de ambos os sexos, com nível intelectual médio. Elas foram distribuídas em dois grupos de 25 sujeitos, um deles com atraso escolar, e o outro grupo apresentando desempenho escolar satisfatório e idade compatível à série cursada. Observou-se através destas técnicas que o rendimento escolar rebaixado nas crianças do grupo com atraso escolar pareceu relacionado a sentimentos de fracasso e a uma autoimagem depreciativa. No grupo de crianças sem atraso escolar predominou, uma melhor utilização dos recursos intelectuais e afetivos, contudo associado a elevado nível de exigência. $\mathrm{O}$ estudo das variáveis afetivas e sua associação ao rendimento escolar puderam favorecer uma compreensão mais aprofundada da maneira como as crianças estão experimentando esta etapa do desenvolvimento.
\end{abstract}

Palavras-chave: rendimento acadêmico; dificuldade de aprendizagem; técnicas projetivas.

\section{Affective Behavior and Academic Achievement}

\begin{abstract}
Using the House-Tree-Person Drawing (HTP) and the Color Pyramid Test (CPT) we aimed at characterizing the affective behavior of 50 children of average intellectual level, male and female, aged from 8 to 12 years. Two groups of 25 subjects each were formed: one presenting school delay and another with satisfactory school performance and age compatible with school grade. The used techniques showed that low school performance among the children with school delay seemed to be related to feelings of failure and a low self-esteem. In the group with no school delay prevailed a better use of affective and intellectual resources, but associated to high demand. The study of affective variables and their association with school performance lead to a deeper understanding of the way the children are experiencing their developing stage.
\end{abstract}

Key words: academic achievement; learning disabilities; projective personality measures.

Na prática clínica, um número elevado de crianças no período inicial de escolarização é trazido para atendimento em função de problemas escolares (Marturano, Magna \& Murtha, 1993). Múltiplos fatores, ambientais e individuais, estão envolvidos no processo de aprendizagem (Boruchovitch, 1994; Fernandez, 1987/1990; Lindahl, 1988; Pain, 1985; Pernambuco, 1992;). Do ponto de vista do ambiente, em uma perspectiva macro, observa-se a influência dos elementos ideológicos, políticos e sociais que dizem respeito à qualidade do ensino, ao papel social da escola e ao preparo dos professores (Focesi, 1990; Oliveira \& Silva, 1990). Em uma perspectiva micro, incluindo o grupo social imediato do indivíduo, tem-se a influência do nível sócio-econômico cultural familiar, o letramento do ambiente familiar, a valorização do ensino formal e a exposição da criança e estresse psicossocial múltiplo.

Do ponto de vista do indivíduo, os fatores internos da criança mais comumente identificados são relativos a aspec-

1 Trabalho desenvolvido como o apoio financeiro da FAPESP. Parte deste material foi apresentado na Dissertação de Mestrado: Crianças com atraso escolar: avaliação psicológica através do HTP e TPC, pela primeira autora, sob orientação da segunda. As demais autoras foram bolsistas de um projeto integrado do CNPq.

2 Endereço: Rua João José Rofrigues de Moraes, 312, City Ribeirão. CEP: 14021-340 Ribeirão Preto - SP. E-mail: oacintra@keynet.com.br tos orgânicos, cognitivos e/ou afetivos. Sabe-se que as alterações orgânicas, como algumas disfunções neurológicas, afetam a capacidade cognitiva. O rebaixamento cognitivo, secundário ou não a outra patologia, definido como retardamento mental implica em "significativa inferioridade do funcionamento intelectual global", bem como na dificuldade adaptativa do indivíduo em "alcançar os padrões esperados para sua idade, seu grupo cultural em áreas como: habilidades sociais e responsabilidades, comunicação, habilidades de vida diária, independência pessoal e auto-suficiência" (DSM-IIIR, p.35). Estes fatores também podem comprometer o bom desempenho acadêmico, acarretando freqüentemente atraso escolar.

Entretanto, muitas crianças sem alterações orgânicas ou deficiência mental apresentam dificuldades de aprendizagem. Esta dificuldade, em geral, tem sido relacionada ao funcionamento afetivo e às funções adaptativas (Feshbach \& Feshbach, 1987; Hall \& Haws, 1989; Lindahl, 1988; Luzuriaga, 1972)

A relação entre o fracasso escolar e o desamparo adquirido, culminando em dificuldades escolares, foi estudada por Nunes (1990). Neste estudo, crianças com dificuldade apresentavam vivências de impotência e fragilidade. No entanto, a experiência de fracasso escolar em muitas crianças não comprometeu sua auto-imagem e a elaboração das dificul- 
dades pareceu possível. Algumas destas utilizaram seus recursos internos para lidar com as experiências de fracasso, não vivenciando depressão.

Diagnosticar as dificuldades afetivas envolve uma complexidade de variáveis. As técnicas de avaliação podem ser instrumentos úteis na compreensão dessas dificuldades. Dentre estas, as técnicas gráficas constituem instrumentos de avaliação dos mais difundidos e utilizados, de aplicação fácil, rápida e baixo custo, que permitem a compreensão dos aspectos estruturais da personalidade, tendo em vista suas características expressivas e projetivas (Ocampo \& Arzeno, 1979/1981).

A bateria gráfica incluindo a representação da casa, árvore e pessoa (HTP) tem sido amplamente estudada e mais recentemente, o Desenho da Figura Humana (DFH) tem constituido-se no instrumento de avaliação gráfico mais abordado, embora haja polêmica na consideração de sua validade. Alguns autores, Gresham (1993), Knoff (1993) e Motta, Little e Tobin (1993) questionaram o seu uso, validade e predição, o que gerou uma série de publicações apontando para as qualidades psicométricas positivas do instrumento, como os estudos de Bardos (1993) e Naglieri (1993). Trabalhos normativos e aplicados com o DFH têm sido também desenvolvidos no contexto brasileiro (Bandeira \& Hutz, 1994; Hutz \& Antoniazzi,1995; Wechsler, 1996).

Numerosos são os trabalhos envolvendo a utilização de "técnicas gráficas" no estudo de crianças com diferentes dificuldades. Com relação a crianças com dificuldades de aprendizagem, Stavrianos (1970) relatou ter encontrado com o HTP, subsídios para o diagnóstico diferencial do déficit de leitura, diagnosticando nas crianças índices sugestivos de retraimento, constrição e dependência. Wagner (1980), em outro estudo, observou sinais de prejuízo na auto-imagem de crianças com dificuldades escolares.

Ao comparar a produção das crianças de classe especial com distúrbios emocionais com crianças que freqüentavam o ensino regular, McNeish e Naglieri (1993) encontraram maior média de sinais associados a distúrbios emocionais no DFH naquelas crianças do grupo especial.

No Brasil, em estudo amplo, Bandeira e Hutz (1994) avaliaram a produção de crianças no DFH e observaram que os escores dos itens evolutivos do DFH mostravam correlação significativa com o rendimento escolar.

Loureiro, Marturano, Linhares, Machado e Silva (1994), estudando crianças com atraso escolar através do HTP, observaram que a produção gráfica e a representação das figuras humanas podem fornecer informações sobre o perfil afetivo. Encontraram no grupo de crianças com atraso escolar indícios de imaturidade, indiferenciação e contenção dos impulsos em si. Consideraram que a procura por atendimento em função do atraso escolar envolve outras dificuldades relativas ao funcionamento afetivo e adaptativo, com prejuízo no processo de amadurecimento.

O Teste das Pirâmides Coloridas de Pfister (TPC) fornece informações sobre os aspectos mais funcionais da personalidade, mostrando ser um método útil, embora menos estudado recentemente (Brauer, 1988; Heiss \& Halder, 1978/
1982; Justo \& Van Kolck, 1985; Kuhn, Gavança, Albernaz, Caorilha \& Marques, 1983).

Souza, Duarte e Cordeiro (1991) utilizaram o TPC na avaliação de grupos de crianças com diferentes níveis de perda auditiva, concluindo que o TPC forneceu dados significativos para a compreensão do funcionamento afetivo de cada subgrupo quando os resultados foram comparados ao padrão normativo.

No presente estudo, objetivou-se a avaliação dos aspectos afetivos associados ao atraso escolar, utilizando as técnicas de avaliação psicológica, HTP e TPC, visando: (a) caracterizar o perfil afetivo das crianças com história de atraso escolar e com nível intelectual médio; (b) comparar o perfil afetivo deste grupo de crianças com o apresentado por um grupo de sujeitos sem atraso escolar.

\section{Material e Método}

\section{Sujeitos}

Foram estudadas 50 crianças, com idade variando entre $8 \mathrm{a} 4 \mathrm{~m}$ a $12 \mathrm{a} 2 \mathrm{~m}$, alunos de $1^{\mathrm{a}}$ à $3^{\mathrm{a}}$ séries da rede pública de ensino, com ingresso na escola aos 7 anos, distribuídas em dois grupos de 25 sujeitos, balanceados com relação ao sexo (14 do sexo masculino e 11 do sexo feminino) e aos anos de escolaridade dos pais. Foram incluídos alunos de 10 escolas de diferentes bairros da cidade. Procurou-se na composição do Grupo 2 incluir crianças que proviessem das mesmas escolas frequentadas pelas crianças do Grupo 1.

Grupo 1. Composto por 25 crianças com história de atraso escolar, cujos pais buscaram atendimento junto ao Ambulatório de Psicologia Infantil do HCFMRP-USP ${ }^{3}$ e com nível intelectual médio nas técnicas de avaliação WISC e Columbia. A faixa etária variou entre $8 \mathrm{a} 4 \mathrm{~m}$ e $12 \mathrm{a} 2 \mathrm{~m}$, sendo a média etária de 9a10m e o grau de instrução médio dos pais e mães correspondente à $4^{\mathrm{a}}$ série do $1^{\circ}$ grau completo.

Grupo 2. Composto por 25 crianças sem história de atraso escolar, frequentando série compatível com sua idade cronológica, sendo indicadas pela professora por apresentarem um rendimento escolar considerado satisfatório e com nível intelectual médio em uma das técnicas de avaliação WISC e Columbia. A faixa etária do grupo variou entre $8 \mathrm{a} 5 \mathrm{~m}$ e $10 \mathrm{a} 7 \mathrm{~m}$, sendo a média etária de $9 \mathrm{a} 1 \mathrm{~m}$ e o grau de instrução médio dos pais e mães correspondente à $4^{\mathrm{a}}$ série do $1^{\circ}$ grau completo.

\section{Procedimento}

\section{Coleta de dados}

As crianças foram avaliadas individualmente em salas com boas condições de iluminação e privacidade em três sessões distintas:

3 Hospital das Clínicas da Faculdade de Medicina de Ribeirão Preto da Universidade de São Paulo. Este Ambulatório presta atendimento psicopedagógico às crianças referidas pela rede de saúde. 
$1^{a}$ Sessão: Rapport e aplicação da Escala de Maturidade Mental Colúmbia (Burgeimester, Blum \& Lorge, 1967);

$2^{a}$ Sessão: Aplicação da Escala Wecshler de Inteligência para Crianças (Poppovic, 1964);

$3^{a}$ Sessão: Aplicação do HTP - Bateria Gráfica CasaÁrvore-Pessoa (Campos, 1969). Após um intervalo, procedeu-se à aplicação do TPC - Teste das Pirâmides Coloridas de Pfister (Amaral, 1978). As crianças do G1 foram avaliadas no ambulatório e as do G2 nas escolas por elas frequentadas.

\section{Tratamento dos dados}

A cotação dos índices do HTP teve por base as proposições de Campos (1969), sendo realizada independentemente pela autora e por outras duas psicólogas com experiência clínica e em avaliação psicodiagnóstica. Considerou-se as cotações de consenso. Objetivando-se a comparação dos grupos, os índices foram classificados considerando o seu significado com base no proposto por diversos trabalhos (Campos, 1969; Hammer, 1981; Van Kolck, 1973; Van Kolck, 1984) sendo 53 índices positivos e 113 índices negativos. Foram classificados positivos aqueles índices tidos como próprios da produção de escolares, os relacionados aos recursos cognitivos ou afetivos, considerados indicadores potenciais para o desenvolvimento. Os índices classificados como negativos foram aqueles considerados inapropriados à produção de escolares, relacionados a prejuízo cognitivo ou afetivo, tidos como sugestivos de atraso ou dificuldade no desenvolvimento. Os índices (166) foram submetidos à análise estatística.

Cada índice identificado recebeu um ponto, positivo ou negativo, permitindo assim pela somatória dos pontos o cálculo dos escores positivo, negativo e global. Para tal, considerou-se a ponderação pelo número total de itens incluídos em cada categoria e acrescentando-se uma constante de forma a evitar respostas negativas.

A comparação entre os Grupos 1 e 2 foi realizada através da análise univariada para medidas repetidas (ANOVA), adotando-se nível de significância $p \leq 0,05$, tomando como base os 166 índices gerais e específicos da técnica gráfica, o Escore Positivo (EP), o Escore Negativo (EN) e o Escore Global (EG).
Utilizou-se também a Análise de Variância Multivariada (MANOVA) para a comparação dos grupos, tomando-se como base os dois escores, positivo e negativo.

Procedeu-se em seguida a comparação dos grupos. Para tal, foi calculado o Índice de Maturidade, com base no tipo de construção das pirâmides, conforme descrito por Justo e Van Kolck (1985). Calculou-se as médias e desvios padrões para os Grupos 1 e 2, e realizou-se análise univariada para medidas repetidas (ANOVA), adotando-se nível de significância $p \leq 0,05$.

Visando a comparação com o padrão teórico (Amaral, 1978), foram trabalhadas as dez cores e as quatro síndromes calculando-se as médias e desvios padrões para cada variável e em seguida aplicou-se o teste "t" de Student.

\section{Resultados}

Considerando-se o objetivo desse estudo, serão apresentadas apenas aquelas variáveis do HTP e do TPC onde podese observar diferenças estatisticamente significativas entre os grupos.

Na Tabela 1, são apresentados os dados relativos ao EP, EN e EG de G1 e G2 no HTP.

Observou-se diferença significativa entre os dois grupos quando analisados os valores relativos ao EP. O G1 apresentou valor médio de EP significativamente menor que o obtido pelo G2. Quanto ao EN, não foram observadas diferenças entre os dois grupos, sendo que ambos apresentaram valores médios muito próximos. A comparação do $\mathrm{EG}$ dos dois grupos apontou para valores menores no G1, em relação aos obtidos pelos sujeitos do G2, no entanto sem significância estatística. Ao considerar-se a comparação de EP e EN por meio da análise estatística (MANOVA), observou-se que os grupos diferenciaram-se em decorrência dos escores positivos.

Assim, a comparação dos escores avaliativos da produção foram sugestivos de poucas diferenças entre os grupos, observando-se uma melhor qualidade na produção dos sujeitos do G2, uma vez que estes apresentaram um maior número de índices com significado positivo.

Na Tabela 2, são apresentados os índices gerais das representações gráficas dos sujeitos no HTP, tomando-se como base o conjunto das cinco representações solicitadas.

Tabela 1 - Comparação da média $(\bar{x})$ e desvio padrão $(s)$ do escore positivo, escore negativo e escore global, obtidos pelas crianças dos Grupos 1 e 2 no HTP.

\begin{tabular}{|c|c|c|c|c|c|c|}
\hline Escore & & Grupo 1 & Grupo 2 & $\mathbf{F}$ & $\mathbf{p}$ & Comparação dos G rupos \\
\hline \multirow[t]{2}{*}{ Positivo $^{1}$} & $\mathrm{x}$ & 39,32 & 45,44 & 7,384 & $0,009 * *$ & $1<2$ \\
\hline & $\mathrm{s}$ & 9,65 & 5,80 & & & \\
\hline \multirow[t]{2}{*}{ Negativo $^{1}$} & $\mathrm{x}$ & 41,88 & 42,76 & 0,282 & 0,598 & $1=2$ \\
\hline & $\mathrm{s}$ & 5,33 & 6,34 & & & \\
\hline \multirow[t]{2}{*}{ Global $^{1}$} & $\mathrm{x}$ & 45,20 & 54,96 & 2,299 & 0,136 & $1<2$ \\
\hline & $\mathrm{s}$ & 25,09 & 20,16 & & & \\
\hline Positivo e Negativo ${ }^{2}$ & - & - & - & 6,777 & $0,003 * *$ & $1 \neq 2$ \\
\hline
\end{tabular}

* $p \leq 0,05 \quad 1 \quad$ ANOVA

** $p \leq 0,01 \quad 2$ MANOVA

$* * * p \leq 0,001$ 
Tabela 2 - Comparação das médias $(\bar{x})$ e desvio padrão $(s)$ dos índices gerais das representações gráficas no HTP que atingiram valores significativos na diferenciação dos Grupos 1 e 20.

\begin{tabular}{|c|c|c|c|c|c|c|}
\hline Variáveis & & G rupo 1 & Grupo 2 & $\mathbf{F}$ & $\mathbf{p}$ & Comparação dos G rupos \\
\hline \multirow[t]{2}{*}{ Produção elaborada } & $\mathrm{x}$ & 0,08 & 0,40 & 70,83 & $0,007 * *$ & $1<2$ \\
\hline & $\mathrm{s}$ & 0,28 & 0,50 & & & \\
\hline \multirow[t]{2}{*}{ Produção empobrecida } & $\mathrm{x}$ & 0,28 & 0,04 & 50,760 & $0,020^{*}$ & $1>2$ \\
\hline & $\mathrm{s}$ & 0,46 & 0,20 & & & \\
\hline \multirow[t]{2}{*}{ Distorção na árvore } & $\mathrm{x}$ & 0,68 & 0,96 & 70,350 & $0,009 * *$ & $1<2$ \\
\hline & $\mathrm{s}$ & 0,48 & 0,20 & & & \\
\hline \multirow[t]{2}{*}{ Traçado apagado, Emend. e Retoc. } & $\bar{x}$ & 0,08 & 0,84 & 660,646 & $0,001 * * *$ & $1<2$ \\
\hline & $\mathrm{s}$ & 0,28 & 0,38 & & & \\
\hline \multirow[t]{2}{*}{ Traçado forte } & $\mathrm{x}$ & 0,64 & 0,88 & 40,114 & $0,048^{*}$ & $1<2$ \\
\hline & $\mathrm{s}$ & 0,49 & 0,33 & & & \\
\hline \multirow[t]{2}{*}{ Traçado médio - normal } & $\mathrm{x}$ & 0,36 & 0,12 & 40,114 & $0,048 *$ & $1>2$ \\
\hline & $\mathrm{s}$ & 0,49 & 0,33 & & & \\
\hline \multirow[t]{2}{*}{ Casa - centro do papel } & $\bar{x}$ & 0,24 & 0,04 & 40,348 & $0,042^{*}$ & $1>2$ \\
\hline & $\mathrm{s}$ & 0,44 & 0,20 & & & \\
\hline \multirow[t]{2}{*}{ Casa - fora do centro } & $\mathrm{x}$ & 0,76 & 0,96 & 40,348 & $0,042^{*}$ & $1<2$ \\
\hline & $\mathrm{s}$ & 0,44 & 0,20 & & & \\
\hline \multirow[t]{2}{*}{ Casa - Iado esquerdo } & $\mathrm{x}$ & 0,64 & 0,36 & 40,083 & $0,049 *$ & $1>2$ \\
\hline & $\mathrm{s}$ & 0,49 & 0,49 & & & \\
\hline \multirow[t]{2}{*}{ Árvore - eixo vertical } & $\bar{x}$ & 0,68 & 0,92 & 40,747 & $0,034^{*}$ & $1<2$ \\
\hline & $\mathrm{s}$ & 0,48 & 0,28 & & & \\
\hline \multirow[t]{2}{*}{ Árvore - abaixo do ponto médio } & $x$ & 0,72 & 0,44 & 40,200 & $0,046^{*}$ & $1>2$ \\
\hline & $\mathrm{s}$ & 0,46 & 0,51 & & & \\
\hline \multirow[t]{2}{*}{ 1a Pessoa - eixo horizontal } & $\mathrm{x}$ & 0,36 & 0,08 & 60,189 & $0,016^{*}$ & $1>2$ \\
\hline & $\mathrm{s}$ & 0,49 & 0,28 & & & \\
\hline \multirow[t]{2}{*}{ 1’a Pessoa - eixo vertical } & $\mathrm{x}$ & 0,64 & 0,92 & 60,189 & $0,016^{*}$ & $1<2$ \\
\hline & $\mathrm{s}$ & 0,49 & 0,28 & & & \\
\hline \multirow[t]{2}{*}{ 1a Pessoa - Iado esquerdo } & $\mathrm{x}$ & 0,68 & 0,36 & 50,486 & $0,023^{*}$ & $1>2$ \\
\hline & $\mathrm{s}$ & 0,48 & 0,49 & & & \\
\hline \multirow[t]{2}{*}{ 2a Pessoa - eixo horizontal } & $\mathrm{x}$ & 0,32 & 0,08 & 40,747 & $0,034^{*}$ & $1>2$ \\
\hline & $\mathrm{s}$ & 0,48 & 0,28 & & & \\
\hline \multirow[t]{2}{*}{ 2a Pessoa - eixo vertical } & $x$ & 0,68 & 0,92 & 40,747 & $0,034^{*}$ & $1<2$ \\
\hline & $\mathrm{s}$ & 0,48 & 0,28 & & & \\
\hline \multirow[t]{2}{*}{ 2a Pessoa - Iado esquerdo } & $x$ & 0,68 & 0,40 & 40,112 & $0,048^{*}$ & $1>2$ \\
\hline & $\mathrm{s}$ & 0,48 & 0,50 & & & \\
\hline \multirow[t]{2}{*}{ 1a Pessoa - muito pequeno } & $\mathrm{x}$ & 0,28 & 0,04 & 50,760 & $0,020^{*}$ & $1>2$ \\
\hline & $\mathrm{s}$ & 0,46 & 0,20 & & & \\
\hline \multirow[t]{2}{*}{ 2a Pessoa - muito pequeno } & $\mathrm{x}$ & 0,28 & 0,04 & 50,760 & $0,020^{*}$ & $1>2$ \\
\hline & $\mathrm{s}$ & 0,46 & 0,20 & & & \\
\hline
\end{tabular}

Em 11 itens, os sujeitos do G1 obtiveram valores médios significativamente maiores que o $\mathrm{G} 2 \mathrm{e}$ em sete itens os sujeitos do G1 obtiveram valores médios significativamente menores que o G2. Os itens mais gerais relacionados à qualidade da produção diferenciaram os grupos de forma significativa, ou seja, em G1 os dados apontaram no sentido de uma produção empobrecida, comparativamente a G2, onde predominou uma produção elaborada. Considerou-se como produção empobrecida figuras esquemáticas pouco características da fase de grafismo de realismo intelectual própria desta faixa etária. Como produção elaborada considerou-se as figuras que apresentavam características próprias desta fase de grafismo ou indícios de maior desenvolvi- mento, como a presença de perspectiva, proporção e adequação do detalhe/todo.

Os demais itens que atingiram significância estatística na diferenciação dos grupos têm seus significados relacionados a uma diversidade de conflitos emocionais.

A presença de distorção no desenho da árvore foi menos freqüente nos sujeitos do G1, apontando para diferenças significativas.

Em três itens relativos ao traçado, os sujeitos do G1 apresentaram valores médios significativamente menores que o G2. Em um item, os sujeitos do G1 obtiveram médias significativamente maiores que os sujeitos do G2 (Traçado Médio-Normal). 
De modo mais geral, as localizações das representações foram classificadas em: centro do papel e fora do centro do papel. Observou-se diferença significativa entre os dois grupos, sendo que os sujeitos do G1 apresentaram valores médios maiores de representações no centro do papel, e apresentaram valores médios menores de representações fora do centro do papel, o que também aponta para um significado positivo. Os desenhos realizados fora do centro tinham localização no lado esquerdo do papel, sugerindo indícios de impulsos agressivos.

Observou-se com relação à representação da árvore, a presença de dois índices que diferenciaram os grupos, ambos relativos à localização do desenho.

Em quatro itens relacionados à localização das figuras humanas, a média obtida pelos sujeitos do G1 foram maiores que as obtidas pelos sujeitos do G2. Pode-se observar que os sujeitos do G1 apresentaram valores médios maiores para desenhos na posição horizontal e para figuras no lado esquerdo do papel nas duas figuras humanas. Por outro lado, os sujeitos do G2 mantiveram a posição da folha proposta pelo examinador, colocadas na posição vertical.

Com relação ao tamanho dos desenhos, foram significativos apenas os valores médios maiores dos sujeitos do G1 em relação aos do $\mathrm{G} 2$ em dois itens.

Na Tabela 3, são apresentados os dados relativos aos índices específicos das produções gráficas dos sujeitos de G1 e G2 no HTP.

Os sujeitos do G1 apresentaram menores médias para três índices que atingiram valores estatisticamente significativos, índices que sugerem dificuldade dos sujeitos deste grupo nos contatos interpessoais.

Observou-se com relação às figuras humanas dois itens que diferenciaram claramente os dois grupos considerandose a qualidade da elaboração. Os sujeitos do G1 apresenta-

Tabela 3 - Comparação da média $(\bar{x})$ e desvio padrão $(s)$ dos índices específicos relativos ao conteúdo das representações gráficas no HTP que atingiram valores significativos para a diferenciação dos Grupos 1 e 2 .

\begin{tabular}{|c|c|c|c|c|c|c|}
\hline Variáveis & & Grupo 1 & G rupo 2 & $\mathbf{F}$ & $\mathbf{p}$ & Comparação dos G rupos \\
\hline \multicolumn{7}{|l|}{ Casa } \\
\hline \multirow[t]{2}{*}{ Tipo perfil absoluto } & $x$ & 0,12 & 0,40 & 5,444 & $0,024 *$ & $1<2$ \\
\hline & $\mathrm{s}$ & 0,33 & 0,50 & & & \\
\hline \multirow[t]{2}{*}{ Paredes Desconjuntadas } & $\mathrm{x}$ & 0,52 & 0,80 & 4,594 & $0,037 *$ & $1<2$ \\
\hline & $\mathrm{s}$ & 0,51 & 0,41 & & & \\
\hline \multirow[t]{2}{*}{ J anela com vidraças } & $\mathrm{x}$ & 0,24 & 0,56 & 5,731 & $0,021 *$ & $1<2$ \\
\hline & $\mathrm{s}$ & 0,44 & 0,51 & & & \\
\hline \multicolumn{7}{|l|}{ Figuras humanas } \\
\hline \multirow{2}{*}{ Esquema corporal elaborado } & $x$ & 0,20 & 0,60 & 9,600 & $0,003 * *$ & $1<2$ \\
\hline & $\mathrm{s}$ & 0,41 & 0,50 & & & \\
\hline \multirow[t]{2}{*}{ Esquema corporal primitivo } & $\mathrm{x}$ & 0,80 & 0,40 & 9,600 & $0,003^{* *}$ & $1>2$ \\
\hline & $\mathrm{s}$ & 0,41 & 0,50 & & & \\
\hline \multirow[t]{2}{*}{ Olhos em negrito } & $\mathrm{x}$ & 0,24 & 0,68 & 11,616 & $0,001 * * *$ & $1<2$ \\
\hline & $\mathrm{s}$ & 0,44 & 0,48 & & & \\
\hline \multirow[t]{2}{*}{ Boca com um traço só } & $\mathrm{x}$ & 0,32 & 0,68 & 7,147 & $0,010 * *$ & $1<2$ \\
\hline & $\mathrm{s}$ & 0,48 & 0,48 & & & \\
\hline \multirow[t]{2}{*}{ Pescoço reto } & $\mathrm{x}$ & 0,24 & 0,64 & 9,302 & $0,004 * *$ & $1<2$ \\
\hline & $\mathrm{s}$ & 0,44 & 0,49 & & & \\
\hline \multirow[t]{2}{*}{ Traço marcando Cintura } & $x$ & 0,44 & 0,80 & 7,654 & $0,008 * *$ & $1<2$ \\
\hline & $\mathrm{s}$ & 0,51 & 0,41 & & & \\
\hline \multirow[t]{2}{*}{ Cabeleira rala } & $x$ & 0,32 & 0,60 & 4,112 & $0,048 *$ & $1<2$ \\
\hline & $\mathrm{s}$ & 0,48 & 0,50 & & & \\
\hline \multirow[t]{2}{*}{ Nariz curto e pequeno } & $x$ & 0,16 & 0,44 & 4,941 & $0,031 *$ & $1<2$ \\
\hline & $\mathrm{s}$ & 0,37 & 0,51 & & & \\
\hline \multirow[t]{2}{*}{ Braços em negrito } & $x$ & 0,16 & 0,44 & 4,941 & $0,031 *$ & $1<2$ \\
\hline & $\mathrm{s}$ & 0,37 & 0,51 & & & \\
\hline \multirow[t]{2}{*}{ Pés representados } & $\mathrm{x}$ & 0,72 & 0,96 & 5,760 & $0,020 *$ & $1<2$ \\
\hline & $\mathrm{s}$ & 0,46 & 0,20 & & & \\
\hline \multirow[t]{2}{*}{ Diferenciação entre masc. e fem. } & $x$ & 0,80 & 1,00 & 6,000 & $0,018 *$ & $1<2$ \\
\hline & $\mathrm{s}$ & 0,41 & 0,00 & & & \\
\hline \multirow[t]{2}{*}{ Pescoço ausente } & $\mathrm{x}$ & 0,44 & 0,16 & 4,941 & $0,031 *$ & $1>2$ \\
\hline & $\mathrm{s}$ & 0,51 & 0,37 & & & \\
\hline \multirow[t]{2}{*}{ Pés um para cada lado } & $\mathrm{x}$ & 0,80 & 0,52 & 6,000 & $0,018 *$ & $1>2$ \\
\hline & $\mathrm{s}$ & 0,41 & 0,51 & & & \\
\hline
\end{tabular}

* $p \leq 0,05$

$* * p \leq 0,01$

$* * * p \leq 0,001$ 
ram valores médios menores para esquema corporal elaborado e valores médios maiores para esquema corporal primitivo.

Quanto aos aspectos mais específicos do desenho das figuras humanas, observou-se vários itens indicadores de conflito, dificuldade nos contatos interpessoais e sentimentos de impotência, onde os sujeitos do G1 apresentaram médias inferiores às obtidas pelo G2, sendo que em G1 apenas dois itens apresentaram os valores médios significativamente maiores que os apresentados pelos sujeitos do G2, indicativos de conflitos e dificuldade de controle.

São apresentados a seguir os resultados de G1 e G2 obtidos no TPC.

Os indicadores de recursos cognitivos e emocionais podem ser avaliados a partir do tipo de construção, ou seja, do nível formal das pirâmides. Observou-se diferenças significativas entre os dois grupos quando avaliado o índice de maturidade, considerando $p \leq 0,01$. G1 obteve média igual a $1,48(D P=1,81)$ menor que o $\mathrm{G} 2$ que obteve média igual a 2,92 $(D P=1,41)$ a um nível de significância estatística de $p=0,003$, sugerindo uma capacidade de organização espacial menos elaborada e imaturidade emocional.

$\mathrm{Na}$ Tabela 4, são apresentados os dados relativos às cores obtidas através do TPC, destacando-se a produção das crianças do G1 e do G2.

Quando comparados, G1 e G2 apresentaram valores muito próximos para nove das dez cores, com poucas diferenças estatisticamente significativas. Apenas com relação à cor vermelha os dois grupos se diferenciaram, considerando $p \leq 0,05$.
Os dois grupos diferenciaram-se quando os resultados obtidos para cada cor foram comparados aos valores normativos. G1 não apresentou diferenças estatisticamente significativas quando comparado ao padrão teórico. Já G2 mostrou diferenças significativas para as cores preta com $p \leq 0,05$; vermelha com $p \leq 0,01$; e marrom com $p \leq 0,001$, não se observando diferenças estatísticamente significativas para as demais cores. Pode-se observar que os valores obtidos pelo G2 para as cores preta e marrom foram menores do que o esperado, com base no padrão normativo. Estas são cores relativas aos controles mais primitivos. Por outro lado, com relação à cor vermelha, que representa a ligação com o real mediante as forças impulsivas, os valores foram mais elevados.

$\mathrm{Na}$ Tabela 5, são apresentados os dados relativos às Síndromes Cromáticas obtidas através do TPC, destacandose a produção das crianças de G1 e G2.

Com base nas médias dos grupos, observou-se a ausência de diferenças significativas $(p \leq 0,05)$ para as quatro síndromes, pois embora os grupos tenham se diferenciado quanto às médias das cores, estas diferenças quando combinadas não alcançaram valores significativos estatisticamente. Quando os valores obtidos por G1 e G2 foram comparados ao padrão teórico, não foram observadas variações do G1 em relação à norma. Em G2, observou-se diferença estatisticamente significativa quanto à Síndrome de Estímulo, com $p \leq$ 0,05. A elevação, neste caso, deveu-se ao aumento da cor vermelha, caracterizando o modo de receptividade dos sujeitos do G2 aos estímulos externos e a sua forma de manifestação afetiva.

Tabela 4 - Comparação das cores obtidas no TPC a partir da produção das crianças dos Grupos 1 e 2 em relação ao Padrão Teórico (PT).

\begin{tabular}{|c|c|c|c|c|c|c|c|}
\hline \multirow[b]{2}{*}{ Cores } & & \multicolumn{5}{|c|}{ Grupos } & \multirow[b]{2}{*}{ G $2 \times$ PT } \\
\hline & & G 1 & G 2 & & G $1 \times$ G 2 & G 1 x PT & \\
\hline \multirow[t]{2}{*}{$\mathrm{Vd}$} & X & 17,68 & 17,36 & $\mathrm{~T}$ & 0,162 & $-0,183$ & $-0,704$ \\
\hline & $\mathrm{S}$ & 8,76 & 4,54 & $p$ & 0,872 & 0,857 & 0,488 \\
\hline \multirow[t]{2}{*}{$A z$} & X & 16,64 & 15,20 & $\mathrm{~T}$ & 0,845 & 1,364 & 0,166 \\
\hline & $\mathrm{S}$ & 6,01 & 6,03 & $p$ & 0,402 & 0,185 & 0,870 \\
\hline \multirow[t]{2}{*}{$\mathrm{Vm}$} & $x$ & 14,20 & 18,04 & $\mathrm{~T}$ & $-2,241$ & $-0,581$ & 2,983 \\
\hline & $\mathrm{S}$ & 6,89 & 5,09 & $p$ & 0,030* & 0,567 & $0,006 * *$ \\
\hline \multirow[t]{2}{*}{$\overline{V i}$} & $x$ & 10,16 & 12,64 & $\mathrm{~T}$ & $-1,746$ & $-0,770$ & 1,801 \\
\hline & $\mathrm{S}$ & 5,45 & 4,55 & $p$ & 0,087 & 0,449 & 0,084 \\
\hline \multirow[t]{2}{*}{$\mathrm{Am}$} & $x$ & 10,08 & 9,88 & $\mathrm{~T}$ & 0,119 & 0,860 & 0,791 \\
\hline & $\mathrm{S}$ & 6,28 & 5,56 & $p$ & 0,906 & 0,398 & 0,437 \\
\hline \multirow[t]{2}{*}{$\mathrm{Ma}$} & $\mathrm{X}$ & 7,44 & 5,36 & $\mathrm{~T}$ & 0,778 & $-0,605$ & $-5,133$ \\
\hline & $\mathrm{S}$ & 12,89 & 3,55 & $p$ & 0,440 & 0,551 & $0,001^{* * *}$ \\
\hline \multirow[t]{2}{*}{ La } & X & 7,76 & 7,24 & $\mathrm{~T}$ & 0,453 & $-0,281$ & $-0,991$ \\
\hline & $\mathrm{S}$ & 4,27 & 3,83 & $p$ & 0,653 & 0,781 & 0,331 \\
\hline \multirow[t]{2}{*}{$\mathrm{Pr}$} & $\mathrm{X}$ & 6,04 & 4,48 & $\mathrm{~T}$ & 1,080 & 0,031 & $-2,443$ \\
\hline & $\mathrm{s}$ & 6,52 & 3,11 & $p$ & 0,285 & 0,976 & $0,022^{*}$ \\
\hline \multirow[t]{2}{*}{$\mathrm{Br}$} & X & 5,12 & 5,24 & $\mathrm{~T}$ & $-0,116$ & 1,806 & 1,506 \\
\hline & $\mathrm{S}$ & 3,10 & 4,12 & $p$ & 0,908 & 0,083 & 0,145 \\
\hline \multirow[t]{2}{*}{$\overline{\mathrm{Ci}}$} & $\mathrm{X}$ & 5,16 & 3,84 & $\mathrm{~T}$ & 0,923 & 0,871 & $-0,307$ \\
\hline & $\mathrm{S}$ & 6,66 & 2,61 & $p$ & 0,361 & 0,392 & 0,762 \\
\hline
\end{tabular}

$* \quad p \leq 0,05$

$* * p \leq 0,01$

$* * * p \leq 0,001$ 
Tabela 5 - Comparação das Síndromes Cromáticas obtidas no TPC a partir da produção das crianças dos Grupos 1 e 2 em relação ao Padrão Teórico (PT).

\begin{tabular}{|c|c|c|c|c|c|c|c|}
\hline \multirow[b]{2}{*}{ Síndromes } & & \multicolumn{6}{|c|}{ Grupos } \\
\hline & & G 1 & G 2 & & G $1 \times$ G 2 & G 1 x PT & G 2 x PT \\
\hline \multirow[t]{2}{*}{ Normal } & 8 & 48,44 & 50,48 & $T$ & $-0,705$ & $-0,225$ & 1,001 \\
\hline & $\mathrm{s}$ & 12,44 & 7,39 & $p$ & 0,484 & 0,824 & 0,327 \\
\hline \multirow[t]{2}{*}{ Estímulo } & $\bar{x}$ & 31,96 & 35,44 & $T$ & $-1,423$ & $-0,020$ & 2,463 \\
\hline & $\mathrm{s}$ & 10,04 & 6,98 & $p$ & 0,161 & 0,984 & $0,021^{*}$ \\
\hline \multirow[t]{2}{*}{ Fria } & $\mathrm{x}$ & 43,96 & 44,84 & $T$ & $-0,300$ & $-0,434$ & $-0,095$ \\
\hline & $\mathrm{s}$ & 11,99 & 8,40 & $p$ & 0,765 & 0,669 & 0,925 \\
\hline \multirow[t]{2}{*}{ Incolor } & $\mathrm{x}$ & 16,40 & 13,56 & $T$ & 1,652 & 1,930 & $-0,034$ \\
\hline & $\mathrm{s}$ & 6,22 & 5,93 & $p$ & 0,105 & 0,066 & 0,973 \\
\hline
\end{tabular}

* $p \leq 0,05$

$* * \quad p \leq 0,01$

$* * * p \leq 0,001$

\section{Discussão}

As crianças com atraso escolar, trazidas para atendimento psicopedagógico, apresentaram tanto no HTP quanto no TPC indícios de poucos recursos elaborativos disponíveis, caracterizando produções empobrecidas e primitivas, aparentemente sugestivas de comprometimento cognitivo. Considerando-se que por meio da avaliação cognitiva não se diagnosticou comprometimento intelectual, pode-se supor que estas crianças estão utilizando precariamente seus recursos cognitivos, funcionando em um nível mais primitivo do ponto de vista evolutivo, provavelmente, em função das dificuldades de ordem afetiva, manifestas enquanto imaturidade. A imaturidade e as dificuldades relativas ao processo de amadurecimento também foram observadas no estudo de Loureiro e cols. (1994).

No grupo de crianças com atraso escolar pode-se verificar a presença de muitos índices no HTP relacionados a dificuldades na construção da identidade. Os sinais encontrados, principalmente com relação ao tamanho muito pequeno, sugerem uma auto-imagem marcada por sentimentos de inferioridade e menos valia, com dificuldade na introjeção dos elementos humanos, que parece estar dificultando o bom desenvolvimento afetivo. Observou-se, também, indícios de insegurança e dificuldade nos contatos interpessoais com vivências internas tipo depressivas, associadas a sentimentos de inferioridade e rejeição. Frente a estas vivências estreitam o seu campo perceptivo e de ação, fechando-se em si, tendendo a evitar o contato com os fracassos pela apatia, dissociação e negativismo.

Estudos de Borges e Loureiro (1990); Marturano, Linhares e Parreira (1993) e Marturano e cols. (1993), embora utilizando diferentes metodologias na caracterização do funcionamento de crianças trazidas para atendimento psicopedagógico, relataram observar fragilidade emocional nestas crianças, com indícios de imaturidade e prejuízo na capacidade adaptativa, o que se assemelha ao observado no presente estudo.

A produção das crianças sem atraso escolar, consideradas pela professora como tendo bom desempenho acadêmico, permitiu a observação de boa capacidade elaborativa tanto no HTP quanto no TPC, com melhor utilização dos recursos intelectuais estando a produção mais compatível com sua idade e com seus recursos cognitivos.

Na produção destas crianças, observou-se figuras humanas com um esquema corporal mais elaborado e recursos de melhor diferenciação do masculino e feminino. Estes dados sugerem que as crianças com bom desempenho escolar estão vivenciando a introjeção das figuras humanas e parentais com maior integração e discriminação, permitindo assim a formação da identidade com maiores recursos de maturidade emocional, sugerindo indícios de um processo de desenvolvimento cognitivo e afetivo mais satisfatório.

Apesar da maior maturidade destas crianças, observouse nas duas técnicas índices de conflitos e de um desenvolvimento afetivo marcado por dificuldades.

As crianças sem queixa escolar apresentaram muitos sinais de um alto nível de exigência interna, com uma necessidade premente de estarem atentas e satisfazerem as demandas externas, mostrando-se muito dependentes das expectativas do meio. Este esforço adaptativo constante associou-se à intensa angústia, estando os recursos potenciais destas crianças canalizados para a execução das tarefas, o que lhes confere características de submissão ao meio e exigência com relação a si.

Com auto-exigência e auto crítica intensa, buscam conter em si seus impulsos e ansiedades, restringindo o contato interpessoal pela superficialidade, fixando-se nas tarefas. No entanto, suas necessidades internas, de agressividade e impulsividade pareceram exercer grande pressão, e em situações de maior envolvimento, manifestam-se enquanto agitação e acting-out.

Segundo Fernandes (1992), a agressividade constitui-se em um elemento fundamental para a aquisição de conhecimento e, possivelmente, é um dos fatores que vem favorecendo o bom desempenho acadêmico das crianças sem atraso escolar, na medida em que a agressividade vem sendo utilizada na incorporação do conhecimento. No entanto, na tentativa de satisfazer as demandas externas, pelo esforço de subordinação, a agressividade, enquanto impulso criativo, passa a ser contida em si, cerceando a criatividade e manifestando-se de forma defensiva.

Nesta faixa etária, as crianças vivenciam o conflito entre a realização versus o sentimento de inferioridade. A análise 
dos dados das crianças dos grupos sugeriram que esta etapa está se fazendo intensamente, com diferenças no modo de vivenciá-las.

As crianças com atraso escolar mostraram prejuízo na expressão dos seus recursos potenciais, tanto intelectuais quanto afetivos. Apresentaram menor rendimento e uma produção empobrecida, com padrões mais estereotipados.

Suas vivências depressivas consomem sua energia produtiva, e este funcionamento pareceu se perpetuar frente a um nível elevado de exigência externa. Assim, poderia se pensar que o fracasso escolar mantém este círculo vicioso, de certa forma consumindo os recursos para responder a ele.

Tal dado aproxima-se do encontrado por Marturano e cols. (1993) que observaram, dentre as crianças com queixa de dificuldades escolares, sinais de imaturidade, atraso no desenvolvimento, disfunção na linguagem e traços de temperamento pouco compatíveis com as exigências escolares. Nessas crianças, observaram dificuldades no enfrentamento, impotência e desamparo frente às demandas do meio, com uma diminuição da capacidade de adaptação e vulnerabilidade a novos fatores estressantes.

No presente estudo, analisando-se as respostas afetivas ao fracasso escolar, pode-se pensar na ação da baixa autoestima, impotência e negativismo enquanto características de personalidade das crianças com atraso. Estas características que pareceram já anteceder a vivência escolar podem estar influenciando a forma como as crianças enfrentam as demandas escolares, ou seja, a sua relação com a escola, influenciando deste modo o fracasso. Neste sentido, as caracteristicas de personalidade descritas acima podem ser consideradas como fatores que influenciam o desempenho acadêmico e o desenvolvimento das crianças de forma semelhante ao observado por Nunes (1990) quanto à relação entre fracasso escolar e desamparo adquirido.

Já as crianças sem atraso escolar, que apresentaram maiores índices de maturidade e de um desenvolvimento compatível com sua faixa etária, apresentaram um rendimento mais satisfatório, com maiores recursos produtivos. Mostraram vivenciar, no entanto, um alto nível de ansiedade decorrente da busca ativa de adaptação social, priorizada sobre suas necessidades internas, favorecendo padrões instáveis nas vivências afetivas, ora construtivas, ora desadaptadas.

O rendimento acadêmico no grupo de crianças com queixa de atraso escolar pareceu ser um indicador do comprometimento afetivo, ao passo que para as crianças sem atraso escolar, o bom rendimento pareceu uma possibilidade de adaptação que não exclui a vivência de dificuldades afetivas.

A avaliação do funcionamento afetivo realizada através das técnicas HTP e TPC forneceu informações relevantes e complementares, diferenciando o perfil afetivo dos dois grupos de crianças estudados. De modo geral, pode-se dizer que o HTP permitiu uma melhor compreensão com relação a forma de utilização dos recursos cognitivos e afetivos das crianças de cada um dos grupos na interação com o meio. $\mathrm{O}$ desenho da figura humana destacou-se, diferenciando os dois grupos por meio de um maior número de ítens a nível de significância estatística.
Estes dados corroboram os encontrados através da avaliação do Desenho da Figura Humana nos estudos de Bandeira e Hutz (1994); Fabry e Bertinetti (1990); Hutz e Antoniazzi (1995); McNeish e Naglieri (1993); Wagner (1980) e Wisniewski e Naglieri (1990); e que também utilizaram-se desta técnica. Nesses estudos, o DFH favoreceu a compreensão e diferenciação de características de personalidade de grupos específicos.

O TPC forneceu um menor número de informações na comparação dos grupos, diferenciando-os pouco entre si e com o padrão normativo. Sua principal contribuição deu-se em relação ao índice de maturidade permitindo a compreensão das diferenças entre os grupos quanto a forma de adaptação à realidade.

Estes dados diferiram dos obtidos por Souza e cols. (1991) que utilizaram o TPC em grupos de crianças com diferentes níveis de perda auditiva, permitindo a diferenciação destes em relação ao padrão normativo.

Frente a esses dados, pode-se pensar que o TPC foi sensível para diferenciar grupos de crianças com diferenças e dificuldades objetivas, como pode ser considerada a surdez e os seus diferentes níveis, não diferenciando, contudo, grupos com características avaliadas de forma subjetiva, como as observadas no presente estudo.

Outro aspecto que merece comentários diz respeito aos critérios de inclusão das crianças com queixas de atraso escolar e sem atraso escolar nos grupos do presente estudo. No grupo com atraso, a situação explícita de procura dos pais por auxílio especializado e as reprovações escolares das crianças referendaram, até certo ponto, a situação de atraso escolar, o que provavelmente tem relação com a maior homogeneidade do grupo quanto ao funcionamento afetivo. No grupo sem atraso escolar, o bom desempenho associado à ausência de reprovações, referendado pela avaliação das professoras, critério este sujeito a maior subjetividade, possivelmente favoreceu uma diversidade de características afetivas.

O padrão de funcionamento escolar esperado, segundo Fernandes (1992), além do rendimento, em geral prioriza a aceitação e submissão às normas. Isso pode ter favorecido a inclusão no Grupo 2 de muitas crianças com estas características, perdendo a dimensão adaptativa das mesmas.

\section{Comentários e Conclusões}

No grupo de crianças com queixa de atraso escolar, predominou a dificuldade para a utilização dos recursos potenciais intelectuais e afetivos, caracterizando um empobrecimento e menor capacidade de elaboração e imaturidade. A dificuldade na formação da identidade, marcada por uma auto-imagem depreciativa, pareceu influenciar a forma destas crianças relacionarem-se com as pessoas e com os conhecimentos e, neste sentido, a escola pode estar enfatizando as dificuldades cognitivas e afetivas, na medida em que perpetua a experiência de fracasso. No grupo de crianças sem queixa de atraso escolar, predominou uma melhor utilização dos recursos intelectuais e afetivos, com maior possibi- 
lidade de elaboração e maturidade. As crianças deste grupo mostraram-se muito identificadas com um elevado nível de exigência e de eficiência, revelando necessidades internas de realização. Pode-se pensar que a escola, na medida em que prioriza a execução das tarefas, o faz independentemente do custo emocional.

Nesse sentido, do ponto de vista da Saúde Mental, o rendimento escolar, em geral considerado como um sinal adaptativo importante, mostrou-se aqui acompanhado de dificuldades afetivas presentes na produção das crianças dos dois grupos.

Considerando-se que a busca de realização é uma necessidade própria das crianças desta faixa etária, cuidar do custo emocional desta busca pode ser uma forma de evitar outras dificuldades emocionais. Para tal, do ponto de vista do desenvolvimento, seria importante que as escolas considerassem não só o rendimento enquanto produto escolar, mas o processo através do qual este rendimento se efetiva.

\section{Referências}

Amaral, F.V. (1978). Pirâmides Coloridas de Pfister. Rio de Janeiro: Centro de Psicologia Aplicada (CEPA).

American Psychiatric Association. (1989). DSM-III-R, Manual de Diagnóstico e Estatística dos Distúrbios Mentais ( $3^{\mathrm{a}}$ ed.). (L.H.S. Barbosa, Trad.) São Paulo: Manole.

Bandeira, D.R. \& Hutz, C.S. (1994). A contribuição dos testes DFH, Bender e Raven na predição do rendimento escolar na primeira série. Psicologia: Teoria e Pesquisa, 10(1), 59-72.

Bardos, A.N. (1993). Human figure drawings: Abusing the abused. School Psychology Quarterly, 8(3), 177-181.

Borges, L.A.C. \& Loureiro, S.R. (1990). O desenho da família como instrumento de avaliação clínica de um grupo de crianças encaminhadas para atendimento psicológico. Arquivos Brasileiros de Psicologia, 42(2), 106-14.

Boruchovitch, E. (1994). As variáveis psicológicas e o processo de aprendizagem: uma contribuição para a psicologia escolar. $P$ sicologia: Teoria e Pesquisa, 10(1),129-39.

Burgeimester, B.B.; Blum, L.H. \& Lorge, I. (1967). Escala de Maturidade Mental de Columbia. Manual de Aplicação. São Paulo: Vetor Editora Psicopedagógica.

Brauer, J.F.O. (1988). O teste das Pirâmides Coloridas de Max Pfister - uma releitura. Tese de Doutorado, Universidade de São Paulo, São Paulo.

Campos, D.M.S. (1969). O teste do desenho como instrumento de diagnóstico da personalidade. Petrópolis: Vozes.

Fabry, J.J. \& Bertinetti, J.A. (1990). A construct validation study of the humam figure drawing test. Perceptual and Motor Skills, 70, 465-66.

Fernandez, A. (1990). A inteligência aprisionada. (I. Rodrigues, Trad.) Porto Alegre: Artes Médicas. (Trabalho original publicado em 1987).

Fernandes, A. (1992, março). Agressividade. Qual o teu papel na aprendizagem? (E. Tavares, Trad). Trabalho apresentado na II Jornada de Estudos Pedagógicos, Porto Alegre, Rio Grande do Sul.
Feshbach, N.D. \& Feshbach, S. (1987). Affective processes and academic achievement. Child Development, 58, 1335-1347.

Focesi, E. (1990). Educação em saúde na escola. O papel do professor. Revista Brasileira de Saúde Escolar, 1(2), 5-9.

Gresham, F.M. (1993). What's wrong in this picture?: Response to Motta et al.'s review of human figure drawings. School Psychology Quarterly, 8(3), 182-186.

Hall, C.W. \& Haws, D. (1989). Depressive symptomatology in learning-disabled and nonlearning-disabled students. Psychology in the Schools, 26, 359-364.

Hammer, E.F. (1981). Aplicações clínicas dos desenhos projetivos. Rio de Janeiro: Interamericana. (Trabalho original publicado em 1971)

Heiss, R. \& Halder, P. (1982). O Teste das Pirâmides de Cores. (L. Growald, Trad.) São Paulo: Vetor. (Trabalho original publicado em 1978)

Hutz, C.S. \& Antoniazzi, A.S. (1995). O desenvolvimento do desenho da figura humana em crianças de 5 a 15 anos de idade: normas para avaliação. Psicologia: Reflexão e Crítica, 8(1), 318.

Justo, H. \& Van Kolck, T. (1985). O Teste das Pirâmides de Cores ( $2^{\mathrm{a}}$ ed.). São Paulo: Vetor.

Knoff, H.M. (1993). The utility of human figure drawings in personality and intellectual assessment: Why ask why? School Psychology Quarterly, 8(3), 191-196.

Kuhn, A.M., Gavança, M.M., Albernaz, P.L.M., Caorilha, H.H. \& Marques, M.I.B. (1983). Do perfil psicológico nas síndromes labirínticas com exame vestibular normal alterado. Acta Awho, 2, 11-15.

Lindahl, N.Z. (1988). Personalidade humana e cultura: aplicações educacionais da Teoria de Erik Erikson. Revista Brasileira de Estudos Pedagógicos, 69(163), 492-509.

Loureiro, S.R., Marturano, E.M., Linhares, M.B.M., Machado, V.L.S. \& Silva, S.R. (1994). Crianças com queixa de dificuldade escolar: avaliação psicológica através de técnica gráfica. Arquivos Brasileiros de Psicologia Aplicada, 46(3/4), 161-182.

Luzuriaga, I. (1972). La inteligência contra si mesma. Buenos Aires: Psique.

Marturano, E.M., Linhares, M.B. \& Parreira, V.L.C. (1993). Problemas emocionais e comportamentais associados a dificuldades na aprendizagem escolar. Medicina, 26(2), 161-175.

Marturano, E.M., Magna, J.M. \& Murtha, P.C. (1993). Procura de atendimento psicológico para crianças com dificuldades escolares: um perfil da clientela. Psicologia: Teoria e Pesquisa, 9(1), 207-26.

McNeish, T.J. \& Naglieri, J.A. (1993). Identification of individuals with serious emotional disturbance using the draw-a-person: Screening procedure for emotional disturbance. Journal of Special Education, 27(1), 115-121.

Motta, R.W.; Little, S.G. \& Tobin, M.I. (1993). The use and abuse of human figure drawings. School Psychology Quarterly, 8(3), 162-169.

Naglieri, J.A. (1993). Human figure drawings in perspective. School Psychology Quarterly, 8(3), 170-176. 
Nunes, A.N.A. (1990). Fracasso escolar e desamparo adquirido. Psicologia: Teoria e Pesquisa, 6(2), 139-154.

Ocampo, M.L.S. \& Arzeno, M.E.G. (1981). O processo psicodiagnóstico e as técnicas projetivas. (M. Felzenszwalb, Trad.) São Paulo: Martins Fontes. (Trabalho original publicado em 1979)

Oliveira, M.L.C.L. \& Silva, M.T.A. (1990). Educação em saúde: repensando a formação de professores. Revista Brasileira de Saúde Escolar, 1(2), 13-19.

Pain, S. (1985). Diagnóstico e tratamento dos problemas de aprendizagem. Porto Alegre: Artes Médicas.

Pernambuco, M.C.A. (1992). As funções neuropsicológicas e os distúrbios de aprendizagem. Em B.J.L. Scoz, L.M.C. Barone, M.C.M. Campos \& M.H. Mendes (Orgs.), Psicopedagogia contextualização, formação e atuação profissional (pp. 68-77). Porto Alegre: Artes Médicas.

Poppovic, A.M. (1964). Manual da Escala de Inteligência Wechsler para criança. Rio de Janeiro: CEPA.
Souza, R.M., Duarte, F., Cordeiro, J.A. (1991). Desenvolvimento afetivo medido pelo teste das pirâmides coloridas de Pfister e graus de perda auditiva: um estudo exploratório. Estudos de Psicologia, 8(2), 65-101.

Stavrianos, B.K. (1970). Emotional and organic characteristics in drawing of deficient readers. Journal of Learning Disabilities, 10, 488-501.

Van Kolck, O.L. (1973). O desenho da figura humana no estudo de problemas específicos. Boletim de Psicologia, 25(65), 151-81.

Van Kolck, O.L. (1984). Testes projetivos gráficos no diagnóstico psicológico. São Paulo: EPU.

Wagner, R.F. (1980). Human figure drawings of L.D. children. Academic Therapy, 16, 37-41.

Wechsler, S.M. (1996). O desenho da figura humana: avaliação do desenvolvimento cognitivo infantil. Campinas: Editorial Psy.

Wisniewski, J.J. \& Naglieri, J.A. (1990). Validity of the draw-aperson: a quantitative scoring system with the WISC-R. Journal of Psychoeducation Assessment, 7, 346-51. 